\title{
A Computer Algorithm for Automatic Beam Steering
}

\author{
Erika Drennan \\ Fermi National Accelerator Laboratory \\ P.O. Box 500, Batavia, Illinois 60510
}

June 1992 


\section{Disclaimer}

This report was prepared as an account of work sponsored by an agency of the United States Government. Neither the United States Government nor any agency thereof, nor any of their employees, makes any warranty, express or implied, or assumes any legal liability or responsibility for the accuracy, completeness, or usefulness of any information, apparatus, product, or process disclosed, or represents that its use would not infringe privately owned rights. Reference herein to any specific commercial product, process, or service by trade name, trademark, manufacturer, or otherwise, does not necessarily constitute or imply its endorsement, recommendation, or favoring by the United States Government or any agency thereof. The views and opinions of authors expressed herein do not necessarily state or reflect those of the United States Government or any agency thereof. 


\title{
A Computer Algorithm for Automatic Beam Steering
}

\author{
Erika Drennan
}

May 1, 1992 
Beam steering is done by modifying the current in a trim or bending magnet. If the current change is the right amount the beam can be made to bend in such a manner that it will hit a swic or BPM downstream from the magnet at a predetermined set point. Although both bending magnets and trim magnets can be used to modify beam angle, beam steering is usually done with trim magnets. This is so because, during beam steering the beam angle is usually modified only by a small amount which can be easily achieved with a trim magnet. Thus in this note, all steering magnets will be assumed to be trim magnets. There are two ways of monitoring beam position. One way is done using a BPM and the other is done using a swic. For simplicity, beam position monitoring in this paper will be referred to being done with a swic. In either case whether trim or bending magnets, and BPM's or swic's are used in a particular beam steering situation, all information in this note will hold.

Beam steering can be done manually by changing the current through a trim magnet and monitoring the position of the beam downstream from the magnet with a swic. Alternatively the beam can be positioned automatically using a computer which periodically updates the current through a specific number of trim magnets. The purpose of this note is to describe the steps involved in coming up with such a computer program. There are two main aspects to automatic beam steering. First a relationship between the beam position and the bending magnet is needed. Secondly a beamline setup of swics and trim magnets has to be chosen that will position the beam according to the desired specifications. A simple example will be looked at that will show that once a mathematical relationship between the needed change of the beam position on a swic and the change in trim currents is established, a computer could be programmed to calculate and update the trim currents.

\section{Example 1:}

Given a series of trims and swics in alternating order, as in Fig. 1, it is clear that $\Delta \mathrm{S}_{1}$ (position change on $\mathrm{S}_{1}$ ) can only be achieved by changing the current $\Delta \mathrm{I}_{1}$ in $\mathrm{T}_{1} . \Delta \mathrm{S}_{2}$ is affected by $\Delta \mathrm{I}_{1}$ and $\Delta \mathrm{I}_{2}$ and so on.

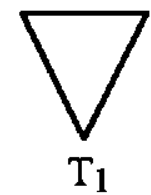

$\mathrm{T}_{1}$
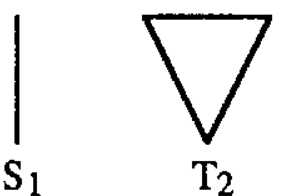

$\mathrm{T}_{2}$

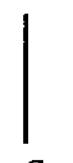

$\mathrm{S}_{2}$

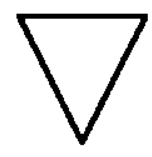

$\mathrm{T}_{3}$

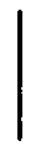

$\mathrm{S}_{3}$

Figure 1: Set-up for Example 1.

If it is assumed that the swic positions are proportional to the trim currents then a set of equations, Equation 1, can be written that describes the effect of changing trim currents on changes in swic positions $\Delta \mathrm{S}$ 's:

$$
\begin{aligned}
& \Delta \mathrm{S}_{1}=\mathrm{C}_{11} \Delta \mathrm{I}_{1}+0 \Delta \mathrm{I}_{2}+0 \Delta \mathrm{I}_{2} \\
& \Delta \mathrm{S}_{2}=\mathrm{C}_{12} \Delta \mathrm{I}_{1}+\mathrm{C}_{22} \Delta \mathrm{I}_{2}+0 \Delta \mathrm{I}_{3} \\
& \Delta \mathrm{S}_{3}=\mathrm{C}_{13} \Delta \mathrm{I}_{1}+\mathrm{C}_{23} \Delta \mathrm{I}_{2}+\mathrm{C}_{33} \Delta \mathrm{I}_{3}
\end{aligned}
$$


In matrix notation:

$$
\Delta \mathbf{S}=\mathbf{C} \Delta \mathbf{I}
$$

Now a matrix equation has been found that solves for $\Delta \mathbf{S}$ if $\Delta \mathbf{I}$ is known. Of course during automatic beam steering, $\Delta \mathbf{I}$ has to be solved for from a knowledge of $\Delta \mathbf{S}$. This is possible if $\mathbf{C}$ is square and nonsingular, $|\mathbf{C}| \neq 0$. The inverse of $\mathbf{C}$ can be found and $\Delta \mathrm{I}$ 's can be solved for :

$$
\Delta I=C^{-1} \Delta S
$$

Equation 3 tells us that for any desired change in beam position on the swics $\Delta \mathbf{S}$, a $\Delta \mathbf{I}$ can be calculated that will cause the beam to move $\Delta S$ amount. This solution would be exact, except for possible inaccuracies in the elements of $\mathbf{C}$. If $\mathbf{C}$ is singular, which would indicate more then one swic for each trim, a matrix still exists to solve for $\Delta \mathbf{I}$ when $\Delta \mathbf{S}$ is known. These situations, which uses least squares approximations, will be discussed later.

\section{Transport Matrices}

It is clear now that if the elements of the $\mathbf{C}$ matrix can be solved for, a computer can be used to periodically solve $\mathrm{C}^{-1} \Delta \mathrm{S}$ and update the currents in the trims. It seems to make sense that the elements of $\mathbf{C}$ depend on what kind of beamline elements occupy the space between the swics and the trims. For example, if only free space is between $T_{1}$ and $S_{1}$ then $C_{11}$ in Example 1 is given by $d_{1} a_{1}$ where $d_{1}$ is the distance between $S_{1}$ and $T_{1}$ and $a_{1}$ is a constant associated with the first trim magnet; its units are $\mu \mathrm{rad} / \mathrm{Amp}$.

If there are other beamline elements, such as quadș, bending magnets and drifts (free space), the elements of $\mathbf{C}$ can be solved for by using the transport matrices of each beamline element. A transport matrix gives the relationship between the beam at one point in the beamline and the beam at another point in the beamline. A beam traveling into the $\mathrm{z}$ direction can move off center in both the $\mathrm{x}$ and $\mathrm{y}$ directions. Although a beam steering algorithm should correct errors in both the $\mathrm{x}$ and the $\mathrm{y}$ directions, only the $\mathrm{x}$ direction will be looked at here. Once beam positioning and transport matrices are understood in the $\mathrm{x}-\mathrm{z}$ plane, positioning the beam in the $\mathrm{y}-\mathrm{z}$ plane follows the same rules and it will be discussed later.

A beam can be described by two quantities $\mathrm{x}$ and $\mathrm{x}$, where $\mathrm{x}$ is the displacement of the beam from a center and $\dot{x}=d x / d z$ gives the angle at which the beam is traveling with respect to the horizontal. This is illustrated in Figure 2. 


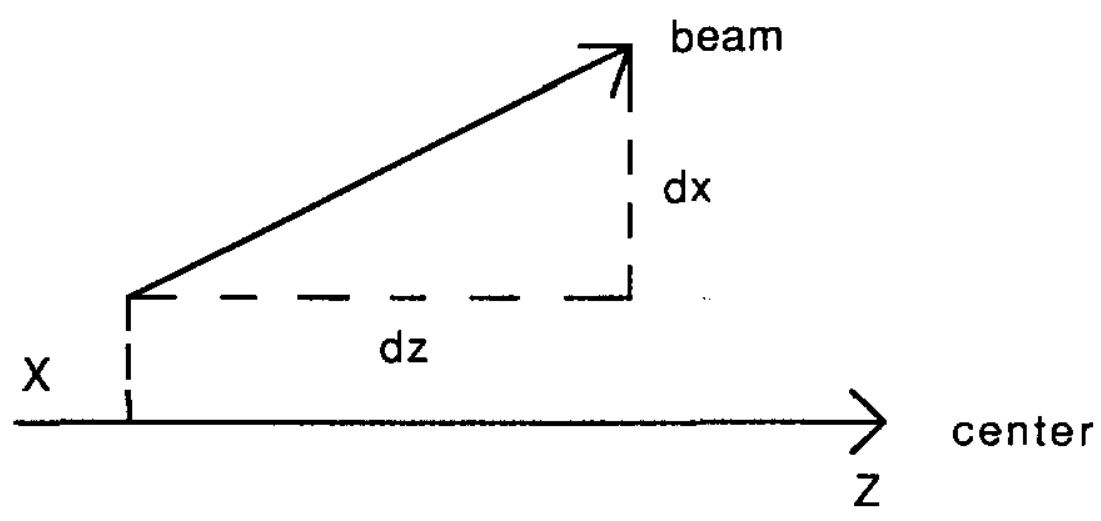

Figure 2: Definitions of $\mathrm{x}$ and $\dot{\mathrm{x}}$

Transport matrices relate $(x, \dot{x})$ at one point in the beamline to $(x, \dot{x})$ at another point in the beamline. For example one can look at the beam at point 1 and at point 2 in a beamline, as shown in Figure 3, and find that a transport matrix exists, Equation 4, which depends on the beamline elements between point 1 and 2 , and describes the beam at point 2 in terms of the beam at point 1 .

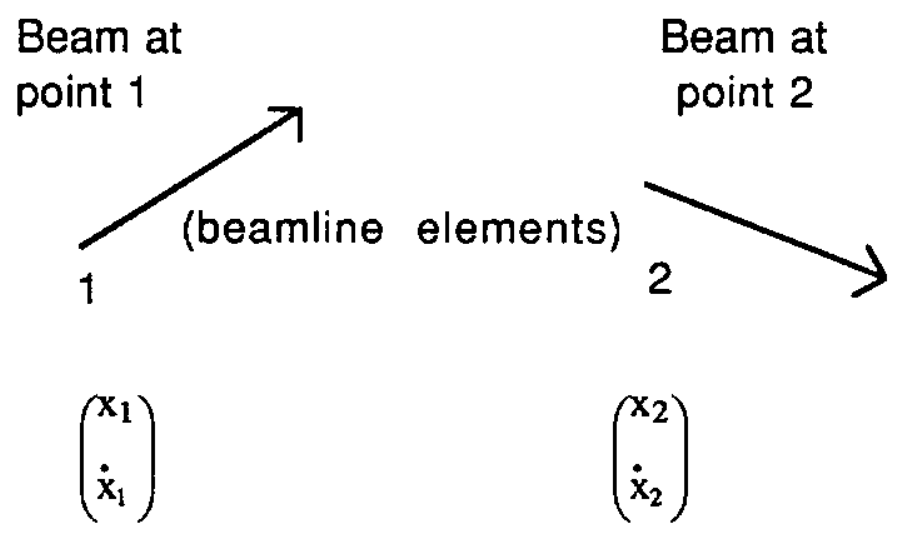

Figure 3: Beam at two points in the beamline.

$$
\left(\begin{array}{l}
\mathrm{x}_{2} \\
\dot{\mathrm{x}}_{2}
\end{array}\right)=\mathrm{T}\left(\begin{array}{l}
\mathrm{x}_{1} \\
\dot{\mathrm{x}}_{1}
\end{array}\right)=\left(\begin{array}{ll}
\mathrm{T}_{11} & \mathrm{~T}_{12} \\
\mathrm{~T}_{21} & \mathrm{~T}_{22}
\end{array}\right)\left(\begin{array}{l}
\mathrm{x}_{1} \\
\dot{x}_{1}
\end{array}\right)
$$

Each beamline element, such as drifts and quads, have a transport matrix that relates the beam at the beginning of that element to the beam at the end. To get the transport matrix $\mathbf{T}$ from point 1 to point 2 , the individual transport matrices of each beamline element between 1 and 2 have to be multiplied. Transport matrices for beamline elements are explained in Reference 1. Since the most commonly used elements in the beamlines are drifts and quads, the transport matrices for these are included in this note. 
The transport matrix for a drift is:

$$
\left(\begin{array}{l}
\mathrm{x}_{2} \\
\dot{x}_{2}
\end{array}\right)=\left(\begin{array}{ll}
1 & \mathrm{~L} \\
0 & 1
\end{array}\right)\left(\begin{array}{l}
\mathrm{x}_{1} \\
\dot{\mathrm{x}}_{1}
\end{array}\right)
$$

The derivation of this matrix is easy to understand by looking at Figure 4 .
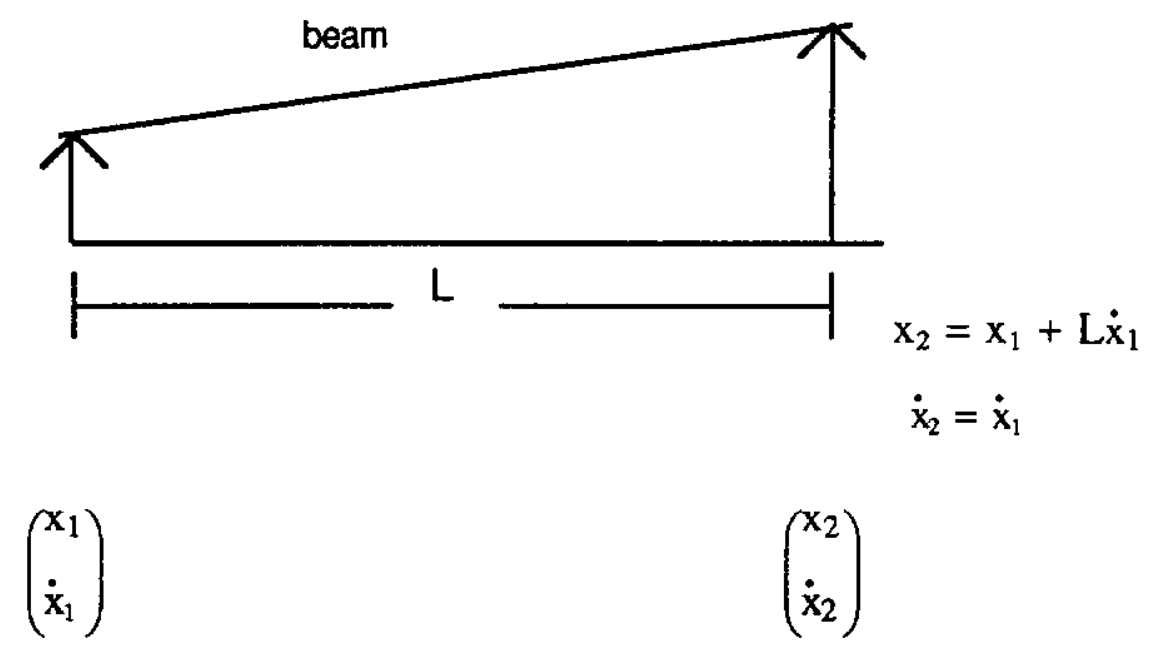

Figure 4: Beam in a drift.

The transport matrix for a quad is more complex and it depends on whether the quadrupole is used for focusing or defocusing. Equation 6 gives the transport matrix for a quadrupole that is focusing in the horizontal $x$-plane. A vertically (y-plane) focusing quadrupole has the two diagonal submatrices interchanged [2].

$$
\left(\begin{array}{l}
\mathrm{x}_{2} \\
\dot{\mathrm{x}}_{2} \\
\mathrm{y}_{2} \\
\dot{y}_{2}
\end{array}\right)=\left(\begin{array}{cccc}
\cos k_{q} L & \frac{1}{k_{q}} \sin k_{q} L & 0 & 0 \\
-k_{q} \sin k_{q} L & \cos k_{q} L & 0 & 0 \\
0 & 0 & \cosh k_{q} L & \frac{1}{k_{q} \sinh k_{q} L} \\
0 & 0 & k_{q} \sinh k_{q} L & \cosh k_{q} L
\end{array}\right)\left(\begin{array}{l}
\mathrm{x}_{1} \\
\dot{\mathrm{x}}_{1} \\
\mathrm{y}_{1} \\
\dot{\mathrm{y}}_{1}
\end{array}\right)(6)
$$

where: $\quad \mathrm{L}=$ the effective length of the quadrupole in meters.

$a=$ the radius of the aperture in $\mathrm{cm}$.

$B_{0}=$ the field at the radius $\mathrm{a}$ in $\mathrm{kG}$.

$k_{q}^{2}=\left(B_{0} a\right)\left(1 / \mathrm{B}_{0} \rho_{0}\right)$, where $\left(B \rho_{0}\right)=$ the magnetic rigidity (momentum) of the central trajectory.

NOTE: In the equation for $k_{q}, \rho_{0}$ is the momentum of the beam. For example, if $L=5 \mathrm{~m}$, $a=5 \mathrm{~cm} \mathrm{~B} B_{0}=5 \mathrm{kG}$ and $\rho_{0}=600 \mathrm{GeV} / \mathrm{C}$, then

$$
k_{q}^{2}=\frac{10}{5} \frac{1}{10 \times 600 \times 10^{9} / 3 \times 10^{8}}=.0001
$$


The only other very commonly used beamline element is a dipole. When writing transport matrices, for the purpose of beam steering, dipoles can be treated as drifts. The following example shows how in a beamline, with several beamline elements between a swic and a trim, the transport matrix between the trim and the swic would be calculated.

\section{Example 2:}

Given the beamline:

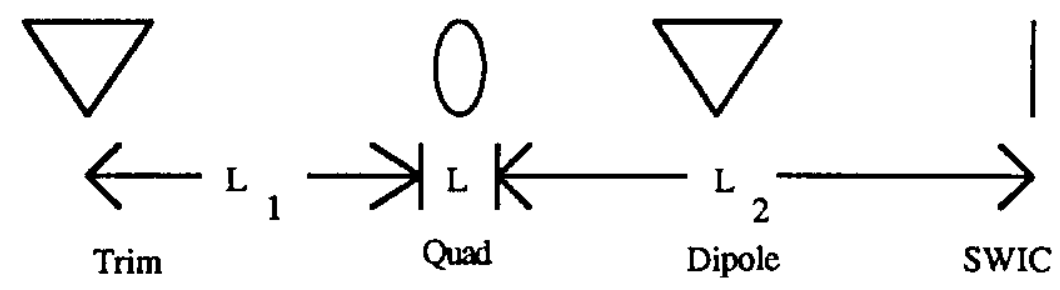

Point 1

Point 2

Figure 5: Setup for Example 2.

The transport matrices of the elements are multiplied:

$$
\left(\begin{array}{l}
x_{2} \\
\dot{x}_{2}
\end{array}\right)=\left(\begin{array}{cc}
1 & L_{2} \\
0 & 1
\end{array}\right)\left(\begin{array}{cc}
\cos k_{q} L & \frac{1}{k_{q}} \sin k_{q} L \\
-k_{q} \sin k_{q} L & \cos k_{q} L
\end{array}\right)\left(\begin{array}{ll}
1 & L_{1} \\
0 & 1
\end{array}\right)\left(\begin{array}{l}
x_{1} \\
\dot{x}_{1}
\end{array}\right)
$$

To come up with the transport matrix between 1 and 2 :

$$
\left(\begin{array}{l}
\mathrm{x}_{2} \\
\dot{\mathrm{x}}_{2}
\end{array}\right)=\left(\begin{array}{ll}
\mathrm{T}_{11} & \mathrm{~T}_{12} \\
\mathrm{~T}_{21} & \mathrm{~T}_{22}
\end{array}\right)=\mathrm{T}\left(\begin{array}{l}
\mathrm{x}_{1} \\
\dot{\mathrm{x}}_{1}
\end{array}\right)
$$

From Example 2 it can be seen that if more swics and more trims followed, a transport matrix $\mathbf{T}$ could be found between each. Now that an equation which gives the relationship of the beam between a swic and a trim has been found, the only task that remains is coming up with a $\mathbf{C}$ matrix that relates the required change in beam position on the swic to the change in trim current. For this we replace the absolute position and angle in Equation 8 by the changes in position and angle. The result is Equation 9:

$$
\left(\begin{array}{c}
\Delta \mathrm{x}_{2} \\
\Delta \dot{\mathrm{x}}_{2}
\end{array}\right)=\left(\begin{array}{cc}
\mathrm{T}_{11} & \mathrm{~T}_{12} \\
\mathrm{~T}_{21} & \mathrm{~T}_{22}
\end{array}\right)\left(\begin{array}{c}
\Delta \mathrm{x}_{1} \\
\Delta \dot{\mathrm{x}}_{1}
\end{array}\right)
$$

In Equation $9 \Delta \dot{x}_{1}=a \Delta I$, which means that the change in beam angle at the trim equals a constant times the change in trim current. The constant $\mathrm{a}$ is in $\mu \mathrm{rad} / \mathrm{A}$ and is a characteristic constant of the magnet. $\Delta \mathrm{x}_{1}$ is not relevant since the beam position at point 1, Fig. 5 , can not be changed with the trim. Therefore the assumption is made that $\Delta \mathrm{x}_{1}=0$. As a result, to get an 
equation that relates the change in beam position on the swic to the change in trim current, only the $\mathrm{T}_{12}$ element of the $\mathrm{T}$ matrix is needed.

Therefore,

$$
\begin{aligned}
\Delta \mathrm{x}_{2} & =\mathrm{T}_{12} \text { a } \Delta \mathrm{I}_{1} \\
\Delta \mathrm{x}_{2} & =\mathrm{C}_{11} \Delta \mathrm{I}_{1}
\end{aligned}
$$

In the same way as Equation 10 has been derived, a $\mathbf{C}$ matrix in the form of Eq. 1 can be constructed for a beamline that has several swics and trims with beamline elements between each swic and trim.

\section{Beam Steering Algorithms}

Now that a matrix such that $\Delta \mathbf{x}=\mathbf{C} \Delta \mathbf{I}$ can be derived, an algorithm can easily be constructed that will continuously update the currents in the trims to correct for possible errors in the beam positions. Although the entire program can be done through the central VAX computer, doing this lengthens the time that it takes to update the trim magnets. In order to reduce the time as much as possible, this algorithm has two parts. Part 1 is the initialization, which is performed at the VAX, part 2 is the actual updating of the trims which can be done with a microprocessor that is in close proximity to the trim magnet whose current has to be updated.

\section{Initialization (at the VAX)}

1. Get a list of beamline elements between each swic and each trim.

2. Construct transport matrix between each swic and trim.

3. Write $\mathbf{C}$ matrix, where $\Delta \mathbf{x}=\mathbf{C} \Delta \mathbf{I}$ and $\Delta \mathbf{x}$ is the desired change in beam position with respect to "center".

4. Calculate $\mathbf{C}^{-1}$

NOTE: As long as beamline elements are not changed, $\mathbf{C}$ and $\mathbf{C}^{-1}$ are also unchanged.

\section{At the Microprocessor}

5. Get $\Delta \mathbf{x}$ from each swic.

6. Using $C^{-1}$ get $\Delta \mathrm{I}$ needed for all trims to fix swic settings.

7. Go to step 5. Repeat steps 5-7 until all swic readings are within specified tolerance.

The just presented algorithm will work well for a setup in which there are the same number of swics as trims, such as the setup in Example 1. As one can recall, $\mathbf{C}$ is square and nonsingular, therefore $\mathbf{C}^{-1}$ exists.

But in some beamline setups $\mathbf{C}$ is no longer square and $\mathbf{C}^{-1}$ does not exist. This happens when there are several swics for each trim such as shown in Fig. 6. Although in this case it is impossible to position the beam to a set point on all three swics with the use of just one trim magnet, it is possible 


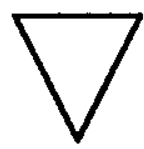

$\mathrm{T}_{1}$

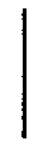

$S_{1}$

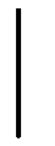

$S_{2}$

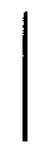

$\mathrm{S}_{3}$

Figure 6: Beam steering setup with several swics for each trim.

to come up with least squares approximation that would minimize the error between the beam position on the swic and the set points. The controller's algorithm would still have two parts, one at the VAX and the other one at the microprocessor. An algorithm for such a setup follows.

\section{Initialization (at the Vax)}

1. Get a list of beamline elements between each swic and each trim

2. Construct a transport matrix between each swic and trim magnet

3. Write $\mathbf{C}$ matrix, where $\Delta \mathbf{x}=\mathbf{C} \Delta \mathbf{I}, \Delta \mathbf{x}$ is the error in beam position with respect to the "center" and $\Delta \mathbf{I}$ is the required current change in the trim magnets.

Note: If, for any trim, there is more than one swic, then the $\mathrm{C}$ matrix is $\mathrm{m} \times \mathrm{n}$ where $\mathrm{m}>\mathrm{n}, \mathrm{m}=$ number of swics and $\mathrm{n}=$ number of trims. It is assumed that $\mathbf{C}$ will have full rank $\mathrm{n}$ since, for every trim, there is at least one swic. Since the $\mathbf{C}$ matrix is not square due to there being more than one swic for each trim, $\Delta I=C^{-1} \Delta x$ cannot be calculated. But a least squares fit solution over all $\Delta x^{\prime}$ s can still be found. In order to do this, $\Delta \mathrm{I}=\left(\mathrm{C}^{\mathrm{T}} \mathrm{C}\right)^{-1} \mathbf{C}^{\mathrm{T}} \Delta \mathbf{x}$.

4. Let $\left(\mathbf{C}^{\mathbf{T}} \mathbf{C}\right)^{-1} \mathbf{C}^{\mathrm{T}}$, which is called the pseudoinverse, be denoted as $\mathbf{C}^{+}$. Send $\mathbf{C}^{+}$to the microprocessor.

\section{At the microprocessor}

5. Get $\Delta \mathbf{x}$ from each swic.

6. Using $\mathrm{C}^{+}$get $\Delta \mathbf{l}=-\mathrm{C}^{+} \Delta \mathbf{x}$ needed to set all trims to fix swic readings.

7. Go to 5. Repeat steps 5-7 until all swic readings are within a specified tolerance.

NOTE: In some solutions a weighted least squares solution may be more desirable. One good source of information on weighted least squares approximations is Ref. 2.

\section{Beamline Systems}

It is possible to space the trims and the swics in several different configurations. Not only will the $\mathbf{C}$ matrix be different depending on which configuration is used, it will also affect how accurately the beam can be positioned. In this section, several setups will be looked at.

A simple beamline setup of the form given in Example 1 is shown in Fig. 7. As it has 
already been shown, the $\mathbf{C}$ matrix

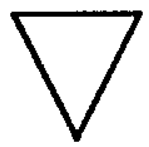

$\mathrm{T}_{1}$

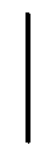

$S_{1}$

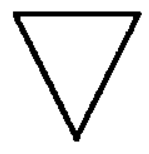

$\mathrm{T}_{2}$

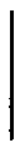

$\mathrm{S}_{2}$

Figure 7: Simple beamline setup.

for this situation is lower triangular and nonsingular. There is one problem with the setup of Fig. 7 and that is that the beam will probably never be straight. It is easy to see that the beam could hit the center on both swics, yet never be straight. An easy way to solve this problem is to move $S_{1}$ and $T_{2}$ very close to each other, where they are at essentially the same place in the beamline. This is shown in Fig. 8. In this configuration $T_{1}$ is used to position the beam at the center point of $T_{2}$ and $T_{2}$ can be used to compensate for any angle $(x)$ in the beam as it enters $T_{2}$.

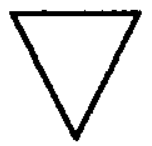

$\mathrm{T}$

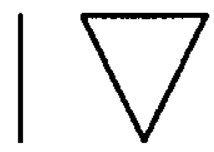

$\mathrm{S}_{1} \quad \mathrm{~T}_{2}$

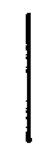

$\mathrm{S}_{2}$

Figure 8: Setup for straight beam.

Another setup to straighten out the beam is shown in Fig. 9. If this setup is used, the C matrix is no longer lower triangular, but it is still nonsingular and its inverse can be easily found. In the case of Fig. 9, the two trims work together to straighten out the beam between the two swics.

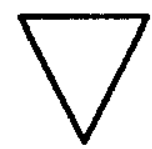

$\mathrm{T}_{1}$

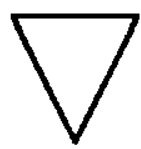

$\mathrm{T}_{2}$

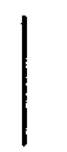

$\mathrm{S}_{1}$

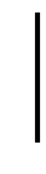

$\mathrm{S}_{2}$

Figure 9: Another setup for straight beam.

To solve for any of these three configurations, the first algorithm of the last section can be used. If more swics are used than trims and it is decided that the beam position error will be minimized using least squares approximation, the second algorithm should be used.

\section{Y-Direction}

Once the $x$-component of the beam has been straightened out, the y component can be straightened out in a similar fashion. In order to bend the beam in the $\pm y$-direction, the trim magnet has to be turned so that the B-field created is in the $\pm x$-direction. There may be many ways of setting up a beamline to correct both $\mathrm{x}$ and $\mathrm{y}$ components of the beam. Only a few will be presented here. 
One way of straightening the y-component of the beam would be to set up another beamline after $x$ has been straightened out and straighten out $y$. This scenario is illustrated in Fig. 10. In this figure the trims used to bend the beam in the horizontal $x$-plane have an $x$ in them, the trims used to bend the beam in the vertical $y$-plane have a $y$ in them.

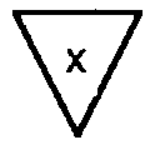

$T_{1}$

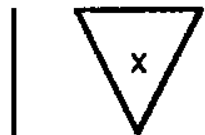

$\mathrm{S}_{1}$

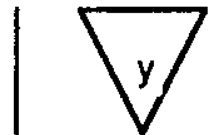

$S_{2}$

T3

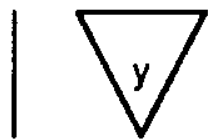

$\mathrm{S}_{3} \quad \mathrm{~T}_{4}$

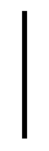

$\mathrm{S}_{4}$

Figure 10: Beam steering in both $\mathrm{x}$ and $\mathrm{y}$ planes.

Although at first look the setup of Fig. 10 may look like the logical choice for beam steering in both the $x$ and $y$ planes, there are two problems with it. One of the problems is inefficient beam use, since two separate beamline setups are needed. The other problem has to do with introducing error into the beam position in the $x$-plane while positioning the beam in the $y$ plane. The way this happens is that the beam may be straight, have no $x$-component at $S_{2}$, but since it still has to travel a long distance while the y component is being corrected, error could be introduced into it by the time it reaches $S_{4}$. If a target is located just downstream of $S_{4}$, the beam may be off in the $\mathrm{x}$-direction.

The problem of introducing error could be overcome by eliminating $S_{2}$ in Fig. 10. If this was done $T_{1}, S_{1}, T_{2}, S_{4}$ could be used to correct the beam in the $x$-direction and $T_{3}, S_{3}, T_{4}, S_{4}$ could be used to correct the beam in the $y$-direction. Using $S_{4}$ twice is possible because all swics provide information regarding the error of the beam position in both $\mathrm{x}$ and $\mathrm{y}$ directions. In fact swics give the beam position in the $x-y$ plane. Since changing the current in $T_{3}$ and $T_{4}$ can in no way affect the $x$-component of the beam, this method would work well to position the beam at the target, but beam usage is still inefficient.

A setup that is quite simple and takes advantage of all the information available on the swic is shown in Fig. 11. In this situation two beam steering algorithms would run simultaneously. One would correct the $x$ component, the other the $y$. The transport matrices in both cases would be written as it has already been explained, although care should be taken in the case of beamline elements such as quads that affect both $\mathrm{x}$ and $\mathrm{y}$ components of the beam differently, that the right submatrix is used in the $x$ and $y$ subsystems.
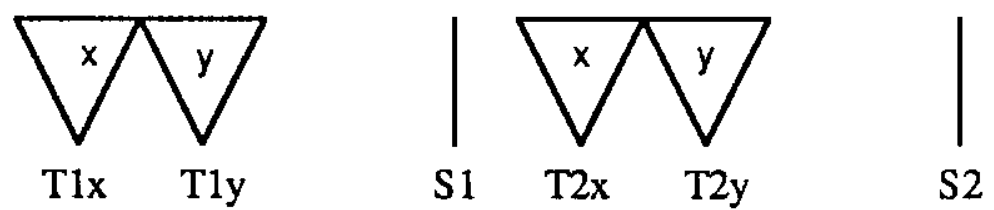

Figure 11: Beam steering in $\mathrm{x}-\mathrm{y}$ planes with using only 2 swics.

For the setup in Fig. 11, first the $\mathrm{x}$ readings of the swics can be corrected, then the $\mathrm{y}$ readings. Due to the long distances between elements in the beamlines, $\mathrm{Tlx}$ and T1y are essentially at the same position and $\mathrm{S} 1, \mathrm{~T} 2 \mathrm{x}, \mathrm{T} 2 \mathrm{y}$ are also at essentially the same position.

Whenever both the $\mathrm{x}$ and $\mathrm{y}$ components of the beam have to be corrected, it is important to completely decouple $\mathrm{x}$ and $\mathrm{y}$. A transport matrix and a $\mathrm{C}$ matrix should be written to correct for the $\mathrm{x}$ component and the algorithm given in previous sections should be followed. Then the $\mathrm{C}$ 
matrix has to be written for the y components and one of the algorithms should be followed. The actual time at which the current in the trim magnets is updated can take place at the same time for $x$ and $y$. Only after both the $x$ and $y$ trims had their current updated should beam positions be read again and the beam positioning algorithms repeated.

\section{Final Notes}

The purpose of the beam steering algorithm was to compensate for small deviations from the center in beam positions. It was assumed that the beam has initially been positioned so that it hits all swics at the desired set point. As a result the $\Delta I$ 's that come out of the program should be small. Therefore it is wise to set limits on how large the needed change in beam position $\Delta \mathbf{x}$ can be and how large a current change the program is allowed to ask for. If either quantity becomes larger than the limit, the autotuning program should stop and manual tuning may be necessary. Also, since positioning the beam at an exact point may take an unnecessarily long time, a tolerance around the setpoint should be included in the program. If the beam position is within this tolerance, the beam steering should be considered a success. It can also help (at least at the initial debugging of the system) if there is a provision in the program to stop it at certain points in the running. This would have to be done through the central VAX.

It has been pointed out already that with the use of a microprocessor, the amount of time that is allowed between updating the $\Delta \mathbf{I}$ 's is decreased. The actual frequency at which $\Delta I$ can be updated, depends mostly on how quickly the trim magnets in the beamline can be driven to their new current values. If the algorithms given in this paper will be used, care has to be taken, that the currents in the trims have reached their new value before another swic reading $\Delta \mathbf{x}$, is used to calculate a new set of $\Delta \mathbf{I}$ 's.

\section{Acknowledgements}

I would like to thank Gaston Gutierrez for sharing with me his knowledge on automatic beam steering and transport matrices. I would also like to thank Robert Joshel and Sam Childress for telling me about their work with automatic beam steering at the switchyard. If these people would not have taken time to talk to me about beam steering, this paper would not have been possible. I'm also grateful to Sam Childress for taking time out of his busy schedule to review the physics calculations. Finally, I would like to thank Terry Grozis for typing the paper, and making all the figures.

\section{$\underline{\text { References }}$}

[1] D.C. Carey, The Optics of Charged Particle Beams, Accelerators and Storage Rings, Vol. 6, Hardwood Academic Publishers, New York (1987).

[2] K.L. Brown and F. Rothacker, D.C. Carey, A. Iselin, Trainsport A Computer Program for Charged Particle Beam Transport Systems, National Technical Information Service, U.S. Dept. of Commerce, Springfield, VA (1992).

[3] W.L. Brogan, Modern Control Theory, Prentice Hall, Inc., Englewood Cliffs, New Jersey (1985). 